\title{
Research and evaluation of the measurement uncertainty with the two-dimensional optical calibration plate
}

\author{
Chang'an $\mathrm{Hu}^{1, *}$, Linghui Kong ${ }^{1}$, Hongmei Ouyang ${ }^{1}$, Shutong $\mathrm{Luo}^{2}$, Fei Lv ${ }^{3}$, and Li Yang ${ }^{1}$ \\ ${ }^{1}$ National Institute of Measurement and Testing Technology, 610021, Chengdu, China \\ ${ }^{2}$ Chengdu University of Technology, 610059, Chengdu, China \\ ${ }^{3}$ Chengdu Normal University, 611130, Chengdu, China
}

\begin{abstract}
As a portable standard, two-dimensional optical calibration plate is playing an increasingly important role in precision industry measurement. Therefore, it is of great scientific significance and obvious socioeconomic benefits to carry out the research of comparator. This paper was based on rich experience with the institute in the field of the geometric measurement technology for a long time, the measurement uncertainty of the optical calibration plate was studied, and the measurement uncertainty of the instrument has been researched. The measurement uncertainty of the error of the calibration plate's setting and marking value was $U=(0.14+0.015 \mathrm{~L}) \mathrm{m} k=2 \mathrm{~L}$ : $\mathrm{mm}$.
\end{abstract}

\section{Introduction}

With the transformation and upgrading of China's manufacturing industry into the fast lane, the current market for processing products of higher quality requirements, processing enterprises have brought efficiency and quality challenges. At present, a new product - two-dimensional optical calibration plate has appeared. As an important portable measurement standard, calibration plate has been used more and more in high precision measurement. In this paper, relying on the research of the geometric measurement technology for a long time, and referring to relevant documents ${ }^{[1-5]}$, the measurement uncertainty of the error of the position marking value of two-dimensional optical calibration plate is studied.

\section{An optical calibration plate}

Optical calibration plate, also known as optical mask plate, is a flat plate with a fixed spacing pattern array. It is necessary to establish the geometric model of camera imaging used some applications, including machine vision, image measurement, photogrammetry and 3D reconstruction, which can correct the errors caused by lens distortion. Furthermore, establishing the geometric model can also determine the conversion relation between physical size and pixels, and the relationship between the three-dimensional geometric position of a point on the surface of a spatial object and position in the image. The geometric model of the camera can be obtained through the calculation of the calibration algorithm by photographing the plate with fixed spacing pattern array, so as to obtain high-precision measurement and reconstruction results.
Calibration plates are often used in the calibration of projection equipment, low-end optical tri-coordinate, etc., or used as mask standard in various kinds of lithography achines. Common calibration plates are shown in FIG. 1 and FIG. 2.

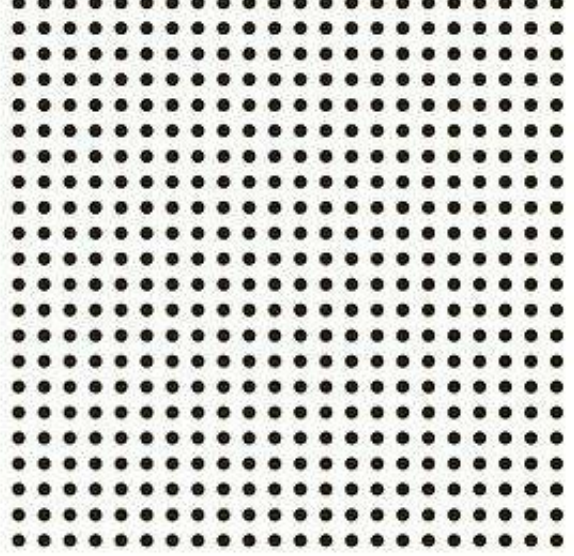

FIG. 1. Solid circle calibration Plate

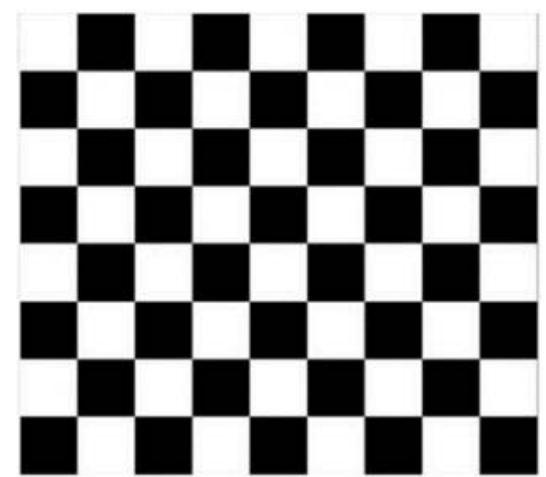

FIG. 2. Checkerboard calibration board

\footnotetext{
* Corresponding author: 569964114@qq.com
} 


\section{Calibration method of calibration plate}

\subsection{The term}

\subsubsection{Origin of coordinates}

For a two-dimensional optical calibration board without designated central dot (or central checkerboard), its coordinate origin is the center of the circle or the checkerboard center at the last row of the calibration board, and the first starting position of the top grid. If there is a center point of the designated center circle (or checkerboard), that point is the origin of coordinates of the calibration board.

\subsection{2 datum axis}

When the $2 \mathrm{~d}$ optical calibration board does not specify the origin of central coordinates, its reference axis is composed of the center line of the circle or the center point of the checkerboard in the last row and the first column of the calibration board, respectively, and the reference axis of the calibration board is $\mathrm{X}$ axis and the reference axis of the $\mathrm{Y}$ axis.

When a two-dimensional optical calibration board has a designated center origin, several center points of the circle or checkerboard in the same row and row with the center origin are connected to form the reference $\mathrm{X}$-axis and the reference $\mathrm{Y}$-axis of the calibration board.

\subsection{Measurement characteristics}

\subsubsection{Error of seat marking value}

The difference between the measured coordinate value and the theoretical coordinate value at the center point of each circle or checkerboard of two-dimensional optical calibration board, $\mathrm{mm}$, and the maximum allowable error is $\pm 0.02 \mathrm{~mm}$.

\subsubsection{Coordinate repeatability}

Under a set of repeatable measurement conditions, the measured precision of the coordinates of the center of the circle or the center point of the checkerboard grid at the farthest vertex Angle from the origin of coordinates is $\mathrm{mm}$. The maximum allowable error is $0.01 \mathrm{~mm}$.

\subsection{Measurement standards and other equipment}

The calibration standard and related measurement parameters are shown in Table 1. The optical tri-coordinate of the standard is shown in FIG. 3.
Table 1. Calibration standard

\begin{tabular}{|c|c|c|}
\hline $\begin{array}{c}\text { Calibration } \\
\text { program }\end{array}$ & $\begin{array}{c}\text { Name of } \\
\text { calibrator used } \\
\text { for calibration }\end{array}$ & $\begin{array}{c}\text { Technical } \\
\text { indicators }\end{array}$ \\
\hline $\begin{array}{c}\text { Error of } \\
\text { coordinates and } \\
\text { coordinate } \\
\text { repeatability }\end{array}$ & $\begin{array}{c}\text { Optical three } \\
\text { two dimensional } \\
\text { coordinate } \\
\text { measuring system } \\
\text { with CCD lens })\end{array}$ & $\begin{array}{c}\text { The indication } \\
\text { error should not } \\
\text { be greater } \\
\text { than: }(0.15+L / 90 \\
0) \mu \text { m } L: m\end{array}$ \\
\hline
\end{tabular}

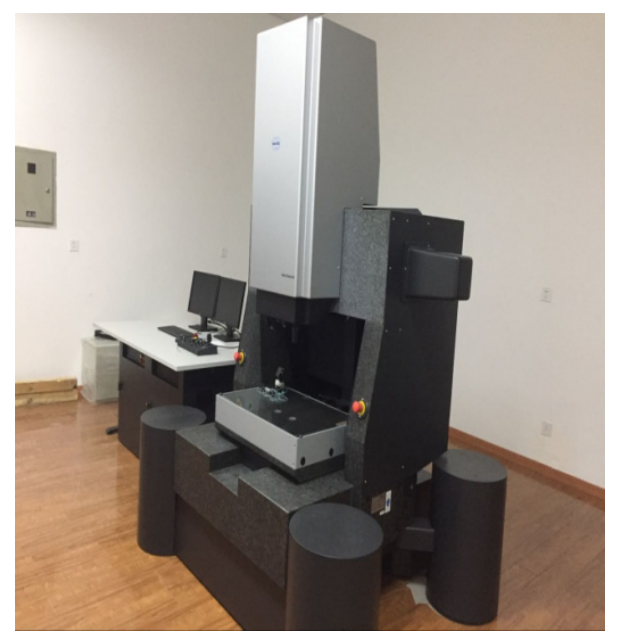

FIG. 3. Optical tri-coordinates

\subsection{Check before calibration}

The newly manufactured or used two-dimensional optical calibration plates shall have models and specifications, factory number, manufacturer or factory name, and the calibration plates shall be free from mold, pitting, scratch, bubble, scratch and other defects. Calibration shall be carried out after determining that there are no factors affecting the metering performance of calibration.

\subsection{Calibration of calibration errors for marked values}

Before calibration, the two-dimensional optical calibration plate should be placed on the optical three-coordinate glass platform at a constant temperature of more than $1 \mathrm{~h}$. The optical three-dimensional (or two-dimensional coordinate measuring system with CCD camera) lens is adjusted to the upside of calibration board, and then selecting the appropriate magnification. Adjusting the focal length until the calibration plate or the edge of the checkerboard can be clearly saw. The sampling of outer contour of round or checkerboard (the 
number of sampling point is not less than 12) was fitted with least squares by the analysis software to calculate the origin of coordinates of the calibration plate. Move the optical three-coordinate lens to the circle or checkerboard close to the origin of coordinates, and then fit again to obtain the value of the center coordinate. Through the same operation, the center coordinate values of each solid circle or checkerboard grid in the same row or row with the origin of coordinates can be measured, and corresponding coordinate systems ( $\mathrm{X}$ and $\mathrm{Y}$ axes) can be established. The coordinate values of each solid circle or checkerboard center of the calibration board are measured by setting an automatic acquisition program. Calculate the error of the seat marking value according to Formula (1-6):

$$
\begin{aligned}
& \Delta X i=X i-X_{\text {cal }} \\
& \Delta Y i=Y i-Y_{\text {cal }} \\
& \triangle X M A X=M A X(\Delta X i) \\
& \triangle Y M A X=M A X(\Delta Y i) \\
& \triangle X M I N=M I N(\Delta X i) \\
& \triangle Y M I N=M I N(\triangle Y i)
\end{aligned}
$$

type:

$\Delta \mathrm{Xi}-\mathrm{X}$ coordinates indicate value error, $\mathrm{mm}$;

$\Delta \mathrm{Yi}-\mathrm{Y}$ coordinates indicate value error, $\mathrm{mm}$;

$\mathrm{Xi}-\mathrm{The}$ actual measurement of the $\mathrm{x}$-coordinate, $\mathrm{mm}$;

$\mathrm{Yi}$ - The actual measurement of the Y-coordinate, $\mathrm{mm}$;

$\mathrm{X}_{\mathrm{cal}}$-The theoretical value of the $\mathrm{X}$ coordinate, $\mathrm{mm}$;

$\mathrm{Y}_{\mathrm{cal}}$ The theoretical value of the $\mathrm{Y}$ coordinate, $\mathrm{mm}$;

\subsection{Variance and sensitivity coefficient}

Each input is independent of each other. According to the propagation rate of uncertainty, it can be concluded that:

$$
u_{2 c}(y)=\sum_{i=1}^{n}\left[c_{i} u\left(x_{i}\right)\right]^{2}
$$

Variance obtained from the measurement model:

$$
u_{2 c}(\Delta L i)=c_{1}^{2} u^{2}\left(L_{i}\right)+c_{2}^{2} u^{2}\left(L_{c a l}\right)
$$

type:

$u\left(L_{i}\right) \quad$ The standard uncertainty component introduced by measurement repeatability;

$u\left(L_{\text {cal }}\right) \longrightarrow$ The standard uncertainty component introduced by the measuring instrument; Sensitivity coefficient:

$$
\begin{aligned}
& c_{1}=\frac{\partial\left(\Delta L_{i}\right)}{\partial L_{i}}=1 \\
& c_{2}=\frac{\partial\left(\Delta L_{i}\right)}{\partial L_{c a l}}=-1
\end{aligned}
$$

conclusion:

$$
u^{2} c(\Delta L i)=u^{2}\left(L_{i}\right)+u^{2}\left(L_{c a l}\right)
$$

\subsection{Repeatability of coordinate measurement}

In the process of calibration of marking value errors, the center of the solid circle or the center of the checkerboard grid at the farthest vertex angle from the origin of coordinates is measured for many times (generally no less than 10 times). The collected data are calculated according to Bessel formula, and the coordinate repeatability of $\mathrm{X}$ and $\mathrm{Y}$ of the calibration plate is respectively calculated, $\mathrm{mm}$.

\subsection{Expression of calibration results}

Calibration shall be carried out in accordance with this specification and a calibration certificate shall be issued. The calibration certificate shall include at least:

A) Title: "Calibration Certificate";

B) Name and address of the laboratory;

C) The location of the calibration (if different from the laboratory address);

D) Unique identification of the certificate (e.g. number), identification of each page and total number of pages;

E) The name and address of the customer;

F) Description and clear identification of the corrected object;

G) The date of the calibration;

$\mathrm{H})$ Identification of the technical specifications on which the calibration is based, including names and codes;

I) Traceability and validity description of the measurement standards used in this calibration;

J) Description of the calibration environment;

K) Signature, position or equivalent identification of the issuer of the calibration certificate or calibration report;

L) The calibration result is only valid declaration for the calibrated object;

M) The declaration of the certificate shall not be partially reproduced without the written approval of the laboratory.

\subsection{Recovery time interval}

It is recommended that the interval of re-schooling should not exceed 12 months.

Note: As the length of the time interval is determined by many factors such as the use of the module, the user, the quality of the module itself, etc., the unit sending the module to school decides the time interval of the time interval according to the actual use. 4 Evaluation and analysis of measurement uncertainty.

\section{Evaluation and analysis of measurement uncertainty}

4.1 Standard uncertainty component $u_{1}$
introduced by calibration board coordinate
measurement repeatability (with the largest 


\section{deviation value of $X$-coordinate and Y-coordinate)}

Under repeated conditions, independent repeated measurements were made for 10 times on the center coordinates of the farthest solid circle or checkerboard grid from the origin of coordinates, and the largest deviation of $\mathrm{X}$ and $\mathrm{Y}$ values was taken. (Table 2)

Table 2. Repeatability test data (unit: $\mathrm{mm}$ )

\begin{tabular}{|c|c|}
\hline The serial number & Measured value \\
\hline 1 & 12.0007 \\
\hline 2 & 12.0007 \\
\hline 3 & 12.0007 \\
\hline 4 & 12.0006 \\
\hline 5 & 12.0006 \\
\hline 6 & 12.0006 \\
\hline 7 & 12.0007 \\
\hline 8 & 12.0007 \\
\hline 9 & 12.0008 \\
\hline 10 & 12.0007 \\
\hline
\end{tabular}

Standard deviation $\mathrm{S}$ of a single measurement can be obtained by Bessel formula:

$$
S=\sqrt{\sum(X i-\bar{X})^{2} / n-1}=0.063 \mu \mathrm{m}
$$

During calibration, the average value of the 10 measurements is taken as the measurement result

$$
u_{1}=\frac{s}{\sqrt{10}}=0.020 \mu \mathrm{m}
$$

\subsection{The standard uncertainty component $\boldsymbol{u}_{2}$ introduced by the standard modulator}

According to the certificate of standard optical 3 coordinates, $U=(0.15+L / 900) \mu \mathrm{m} \quad L: \mathrm{mm}$, including confidence factor $k=2$;

$$
u_{2}=(0.075+0.00056 L) \mu \mathrm{m} L: \mathrm{mm}
$$

\subsection{The uncertainty of temperature linear expansion coefficient $u_{3}$}

The linear expansion coefficient of the two-dimensional optical calibration plate is $10 \times 10^{-6 \circ} \mathrm{C}^{-1}$, and the variation in the half-width interval is $5 \times 10^{-6 \circ} \mathrm{C}^{-1}$. The recommended ambient test temperature is $(20 \pm 2)^{\circ} \mathrm{C}$, and the average deviation is $2^{\circ} \mathrm{C}$. According to the principle of uniform distribution, the following results are obtained:

$$
\begin{gathered}
u_{3}\left(L_{\mathrm{cal}}\right)=L(\mathrm{t}-20) \Delta \alpha / 1.73=L \times 1000 \times 2 \times 5 \times 10^{-6 / 1.73} \\
\mu \mathrm{m}=0.00577 L \mu \mathrm{m}
\end{gathered}
$$

\begin{tabular}{|c|c|c|}
\hline $\begin{array}{l}\text { The } \\
\text { standard } \\
\text { uncertainty } \\
\text { component }\end{array}$ & $\begin{array}{c}\text { Source of } \\
\text { uncertainty }\end{array}$ & $\begin{array}{c}\text { The standard } \\
\text { uncertainty value } u\end{array}$ \\
\hline$u_{1}$ & Measuring & $0.020 \mu \mathrm{m}$ \\
\hline$u_{2}$ & standard & $\begin{array}{c}(0.075+0.00056 L) \\
\mu \mathrm{m}, L: \mathrm{mm}\end{array}$ \\
\hline$u_{3}$ & $\begin{array}{l}\text { Uncertainty of } \\
\text { coefficient of } \\
\text { linear expansion } \\
\text { of temperature }\end{array}$ & $0.00577 L \mu \mathrm{m}, L: \mathrm{mm}$ \\
\hline
\end{tabular}

\subsection{Composite uncertainty}

The uncertainty of synthetic measurement results is shown in Table 3:

Table 3.. List of standard uncertainties

\subsection{Extended uncertainty}

$$
U \approx(0.14+0.015 L) \mu \mathrm{m}, k=2 ; L: \mathrm{mm}
$$

\section{Conclusions}

With the development of the society, more and more precision equipment is used in the field of industrial measurement. At present, the application of two-dimensional optical calibration plate in the field of industrial measurement is increasing year by year. The evaluation of the measurement uncertainty of the standard is helpful to analyze the measurement results.

\section{References}

1. JJF 1071-2010, The Rules for Drafting National Calibration Specification [S].

2. JJF 1001-2011, General Terms in Metrology and Their Definitions[S].

3. JJF 1059.1-2012, Evaluation and Expression of Uncertainty in Measurement[S]. 
4. GB/T 1958-2017 Geometrical product Specifications (GPS)-Geometrical tolerance- Verification prescription[S].

5. JJG 73-2005 Verification Regulation of High-precision Line Scale[S]. 